\title{
Ellis-van Creveld syndrome resulting from segmental uniparental disomy of chromosome 4
}

\author{
S W J Tompson, V L Ruiz-Perez, M J Wright, J A Goodship
}

EDITOR-Our recent report describing the gene mutated in Ellis-van Creveld syndrome (EvC) included a "homozygous" mutation in an affected white subject (RA), ncl-14, whose parents were not known to be related. ${ }^{1}$ The patient's mother and father have died but we have now studied a paternal DNA sample. Analysis of this sample showed that the single nucleotide insertion in exon 7 (910-911 InsA) identified in the patient was not present in her father. The simplest explanation for this would have been a paternally inherited deletion. However, the father was heterozygous for two intronic polymorphisms (IVS7 $+4 \mathrm{~T} \rightarrow \mathrm{C}$ and IVS7 $+10 \mathrm{~A} \rightarrow \mathrm{G}$ ) in the PCR product spanning exon 7 , indicating that this exon was amplified from both paternal chromosomes. Chromosome 4 short tandem repeat (STR) polymorphisms (Research Genetics set 8) were analysed in the samples from the patient and her father and we observed that they did not have an allele in common for D4S2366, the most telomeric marker on the short arm. For all the remaining chromosome 4 short tandem repeat polymorphisms and for nine additional nonchromosome 4 STR polymorphisms the patient and her father shared an allele, making non-paternity extremely unlikely. Additional STR polymorphisms were identified from the physical map of chromosome $4 \mathrm{p}$ at the Stanford Human Genome Center website (http://www-shgc.stanford.edu). The patient was homozygous for all loci telomeric of D4S2632 and there was no paternal allele for D4S1182，D4S1614， D4S412， D4S2925, D4S431, D4S2935, or D4S2366 (fig 1). We conclude that there is segmental uniparental disomy for a region of approximately $30 \mathrm{cM}$ on the short arm of chromosome 4 probably extending to the telomere.

The proband has classical EvC and, therefore, it seems likely that the mitotic recombination that gave rise to this cell line occurred very early in development, the cell line with segmental uniparental disomy contributing the majority of the cells of the inner cell mass. We
School of

Biochemistry and

Genetics, University of

Newcastle, 19/20

Claremont Place,

Newcastle upon Tyne

NE2 4AA, UK

$\mathrm{S}$ W J Tompson

V L Ruiz-Perez

M J Wright

J A Goodship

Correspondence to:

Dr Goodship,

j.a.goodship@ncl.ac.uk

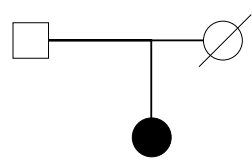

Distance from short Locus arm telomere (cM)

$\begin{array}{clll}0 & \text { D4S2366 } & 1 & 3 \\ 13 & \text { D4S403 } & 1 & 2 \\ 21 & \text { D4S2639 } & 1 & 2 \\ 32 & \text { D4S2397 } & 1 & 1 \\ 40 & \text { D4S2632 } & 1 & 2 \\ 55 & \text { D4S1627 } & 1 & 2 \\ 63 & \text { D4S3248 } & 1 & 1 \\ 70 & \text { D4S2367 } & 2 & 3 \\ 88 & \text { D4S2361 } & 1 & 1 \\ 101 & \text { D4S1647 } & 1 & 2 \\ 126 & \text { D4S2394 } & 1 & 2 \\ 137 & \text { D4S1644 } & 1 & 2 \\ 142 & \text { D4S1625 } & 2 & 3 \\ 156 & \text { D4S1629 } & 1 & 2 \\ 164 & \text { D4S2368 } & 1 & 2 \\ 172 & \text { D4S2431 } & 1 & 1 \\ 179 & \text { D4S2417 } & 1 & 1 \\ 207 & \text { D4S1652 } & 1 & 2\end{array}$

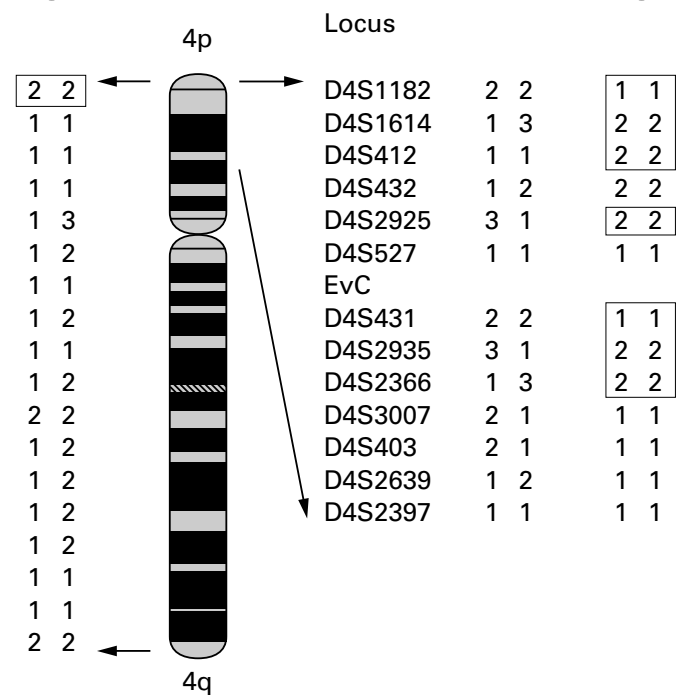

Figure 1 Genotype analysis of chromosome 4 (Research Genetics, left) and telomeric section of chromosome 4p (SHGC right) short tandem repeat polymorphisms in the proband and her father. The distance from the short arm telomere of each right) short tandem repeat polymorphisms in the proband and her father. The distance from the short arm telomere of each (left) and $\gamma-{ }^{32} P$ radiolabelled primers (right). After denaturing, the products were loaded onto a $4 \%$ denaturing polyacrylamide gel and detected using the Genescan (left) or autoradiograph (right) methods. The position of the EvC gene locus is shown with respect to the chromosome 4p STR polymorphisms. Evidence for UPD is indicated by a box in the proband column. 
have not formally tested for low level mosaicism, however. As she did not have any clinical features in addition to those of Ellis-van Creveld syndrome we conclude that this region of chromosome 4 does not harbour any imprinted genes for which paternal expression is essential. This is in keeping with a previous report of uniparental disomy for chromosome 4 detected when chromosome analysis was carried out in the course of investigations in a woman who had had multiple miscarriages. She was found to have iso $(4 p)$ and iso $(4 q) .^{2}$ Molecular studies showed that both derivative chromosomes 4 were of maternal origin. Thus both cases of uniparental disomy involving chromosome $4 \mathrm{p}$ have occurred in females and been of maternal origin.

1 Ruiz-Perez VL, Ide SE, Strom TM, Lorenz B, Wilson D, Woods K, King L, Francomano C, Freisinger P, Spranger S, Marino B, Dallapiccola B, Wright M, Meitinger T, Polymeropoulos MH, Goodship J. Mutations in a new gene in Ellis-van Creveld syndrome and Weyers acrodental dysostosis. Nat Genet 2000;24:283-6.

2 Lindenbaum RH, Woods CG, Norbury CG, Povey S, Rysleckl G. An individual with maternal disomy of Rysleckl G. An individual with maternal disomy of
chromosome 4 and iso $4(\mathrm{p})$, iso $4(\mathrm{q})$. Am $\mathcal{f}$ Hum Genet 1991;49(suppl 285):1582. 